\title{
All-flavor Neutrino Point-like Source Search with the ANTARES Neutrino Telescope
}

\author{
J. Barrios \\ IFIC - Instituto de Física Corpuscular (CSIC - Universitat de València) c/ Catedrático José Beltrán, 2 E-46980 Paterna, \\ Valencia, Spain \\ E-mail: javier.barrios@ific.uv.es

\section{G. Illuminati*} \\ IFIC - Instituto de Física Corpuscular (CSIC - Universitat de València) c/ Catedrático José Beltrán, 2 E-46980 Paterna, \\ Valencia, Spain \\ E-mail:giulia.illuminati@ific.uv.es
}

on behalf of the ANTARES Collaboration

\begin{abstract}
A search for cosmic neutrino sources using the data collected with the ANTARES detector between early 2007 and the end of 2015 is presented. For the first time, all neutrino interactions are considered in a search for point-like sources, instead of only muon neutrino charged current interactions. This is achieved by using a novel reconstruction algorithm for shower-like events in addition to the standard muon track reconstruction. In order to find the clusters of neutrinos from point-like sources over the randomly distributed atmospheric neutrino events, a maximum likelihood ratio approach is followed. The search for astrophysical neutrino sources is performed with four strategies. In the full-sky search, the whole visible sky of ANTARES is scanned to find the most significant cluster of events. In the second approach, the directions of a pre-defined list of known sources which are potential neutrino emitters are investigated. The third search is similar to the full-sky search but restricted to a region around the Galactic Center (GC). Finally, the fourth approach tests the location of Sagittarius A* as an extended source by assuming a Gaussian emission profile of various widths. No significant evidence of cosmic neutrino sources is found. The most significant cluster in the full-sky search is located at $(\alpha, \delta)=\left(343.8^{\circ}, 23.5^{\circ}\right)$ with a post-trial significance of $1.9 \sigma$. Upper limits on the total neutrino flux from the investigated astrophysical candidates are set between $0.60 \times 10^{-8}$ and $2.1 \times 10^{-8} \mathrm{GeV} \mathrm{cm}^{2} \mathrm{~s}^{-1}$. These searches provide the most sensitive limits for a large fraction of the Southern Sky, especially at neutrino energies below $100 \mathrm{TeV}$.
\end{abstract}

35th International Cosmic Ray Conference - ICRC2017

10-20 July, 2017

Bexco, Busan, Korea

${ }^{*}$ Speaker. 


\section{Introduction}

In these proceedings, the results of the latest searches for point-like sources using the ANTARES neutrino telescope [1] are presented. In this work also events identified as electromagnetic or hadronic showers are included in the search for point-like source, unlike previous analyses where only the information from track-like events was exploited. Shower-like events, which contribute about $23 \%$ of all accepted $E^{-2}$ signal events, are reconstructed by means of a new reconstruction algorithm [2], which achieves a median angular resolution between $2^{\circ}$ and $3^{\circ}$ for neutrinos with energies in the $10^{3}-10^{6} \mathrm{GeV}$ range.

\section{Data Sample}

In this analysis, data recorded from Jan 29, 2007 to Dec 31, 2015 are used, for a total livetime of 2423.6 days. The selection of events is performed according to a blinding policy, i.e. using real data scrambled in right ascension (PEs or pseudo-experiments). The selection cuts are chosen so that the neutrino flux needed to make a $5 \sigma$ discovery in $50 \%$ of the pseudo-experiments is minimised when assuming an $E^{-2}$ spectrum. A summary of the different selection criteria for tracks and showers is given below. For the full list of selection cuts, refer to [3].

Muon Track Selection. The muon sample is optimised using three parameters provided by the track reconstruction algorithm - a multi-step fitting procedure that estimates the direction and the position of the muon by means of a maximum likelihood method [4]. Cuts are applied on the reconstructed zenith angle $\left(\cos \theta_{t r}>-0.1\right)$, the estimated angular error $\left(\beta_{t r}<1^{\circ}\right)$ and the parameter that describes the quality of the reconstruction $(\Lambda>-5.2)$. An approximation of the muon energy deposit per unit path length is employed as a proxy of the muon energy [5, 6]. Two additional cuts on energy-related variables are applied to ensure the validity of the energy estimator. The selection yields a total of 7629 neutrino candidates in the track channel.

Shower Selection. Only events not passing the muon track selection are considered in the shower channel. The selection requires that the shower events are reconstructed as up-going or coming from close to the horizon $\left(\cos \theta_{s h}>-0.1\right)$ with constraints on the angular error estimate $\left(\beta_{s h}<30^{\circ}\right)$ and on the interaction vertex, which is required to be reconstructed inside or close to the instrumented volume. The remaining background from mis-reconstructed atmospheric muons is further reduced applying additional selection cuts based on parameters provided by two different shower reconstruction algorithms. A total of 180 neutrino candidates are selected in the shower channel.

\section{Search Method}

A maximum likelihood estimation is performed to identify clusters of cosmic neutrinos from point-like sources over the background of randomly distributed atmospheric neutrinos. The likelihood, by means of signal and background probability density functions (PDFs), exploits the information from different parameters that help to distinguish signal-like clusters from clusters produced by random agglomerations of background events. The used likelihood is defined as 


$$
\log \mathscr{L}_{\mathrm{s}+\mathrm{b}}=\sum_{\mathcal{S}} \sum_{i \in \mathcal{S}} \log \left[\mu_{\text {sig }}^{\mathcal{S}} \mathscr{F}_{i}^{\mathcal{S}} \mathscr{P}_{\text {sig, } \mathrm{i}}^{\mathcal{S}}+\mathscr{N}^{\mathcal{S}} \mathscr{B}_{i}^{\mathcal{S}} \mathscr{P}_{\mathrm{bkg}, \mathrm{i}}^{\mathcal{S}}\right]-\mu_{\text {sig }}
$$

In this equation, $S$ denotes the sample ( $t r$ for tracks, $s h$ for showers), $i$ indicates the event of the sample $S, \mu_{\text {sig }}^{\mathcal{S}}$ is the number of signal events fitted for in the $\mathcal{S}$ sample, $\mathscr{F}_{i}^{\mathcal{S}}$ is a parameterization of the point spread function, i.e. the probability density function of reconstructing the event $i$ at a given angular distance from the true source location, $\mathscr{P}_{\text {sig, }, \mathrm{i}}^{\mathcal{S}}$ is derived from the probability density function of the energy estimator, yielding the probability of measuring the signal with the reconstructed energy of the event $i, \mathscr{N}^{\mathcal{S}}$ is the total number of events in the $\mathcal{S}$ sample, $\mathscr{B}_{i}^{\mathcal{S}}$ is the background rate obtained from the distribution of the observed background events at the declination of event $i, \mathscr{P}_{\mathrm{bkg}, \mathrm{i}}^{\mathcal{S}}$ is the probability density function of the energy estimator for background and $\mu_{\text {sig }}=\mu_{\text {sig }}^{t r}+\mu_{\text {sig }}^{\text {sh }}$ is the total number of fitted signal events.

In the likelihood maximisation, the values of $\mu_{\mathrm{sig}}^{\mathrm{tr}}$ and $\mu_{\mathrm{sig}}^{\mathrm{sh}}$ are left free to vary. Furthermore, the position in the sky of the fitted source is either kept fixed or allowed to be fitted within specific limits depending on the type of search (see Section 4).

In order to estimate the signal likeness of a cluster and determine the significance of any observation, a test statistic is computed as

$$
\mathcal{Q}=\log \mathscr{L}_{\mathrm{s}+\mathrm{b}}-\log \mathscr{L}_{\mathrm{b}},
$$

where $\log \mathscr{L}_{\mathrm{b}}$ is the likelihood defined in equation (3.1) evaluated in the background-only case $\left(\mu_{\text {sig }}^{\mathrm{tr}}=\mu_{\text {sig }}^{\mathrm{sh}}=0\right)$. The behavior of $\mathcal{Q}$ for different signal strengths is determined from pseudoexperiments. In each PE, a number of background events, equal to the number of selected events in data, is distributed on a sky map according to the declination-dependent distribution $\mathscr{B}$, together with signal events injected around a given source position.

\section{Results}

Four different searches for astrophysical neutrino sources are performed. The four approaches together with the respective results are presented below.

\subsection{Full-Sky Search}

The aim of the full-sky search is to find an excess of signal events located anywhere in the ANTARES visible sky, without making any assumption about the source position. To this purpose, the $\mathcal{Q}$-value defined in equation (3.2) is evaluated in steps of $1^{\circ} \times 1^{\circ}$ over the whole scanned region, with the location of the fitted cluster being left free to vary within these boundaries. PEs are performed to obtain the background-only $\mathcal{Q}$ distributions in step of $1^{\circ}$ in declination. From these distributions, the probability to find a $\mathcal{Q}$-value bigger than the one obtained at the cluster position, i.e. the pre-trial p-value, is calculated. The most significant cluster of this search is found at a rightascension of $\alpha=343.8^{\circ}$ and a declination of $\delta=23.5^{\circ}$ with a pre-trial p-value of $3.84 \times 10^{-6}$. The post-trial significance of the cluster, obtained by comparing the pre-trial p-value to the distribution of the smallest p-values found anywhere in the sky when performing the same analysis on many PEs, is found to be $5.9 \%$ or $1.9 \sigma$ (two-sided convention). The upper limits of the highest significant 
cluster in bands of $1^{\circ}$ in declination at a $90 \%$ Confidence Level (C.L.) obtained using the Neyman method [7] are shown in Figure 1. The limits computed in this analysis are set on the total neutrino flux $\left(\Phi_{v_{\mu}}+\Phi_{v_{e}}+\Phi_{v_{\tau}}\right)$, assuming the equipartition at Earth of the three neutrino flavours.

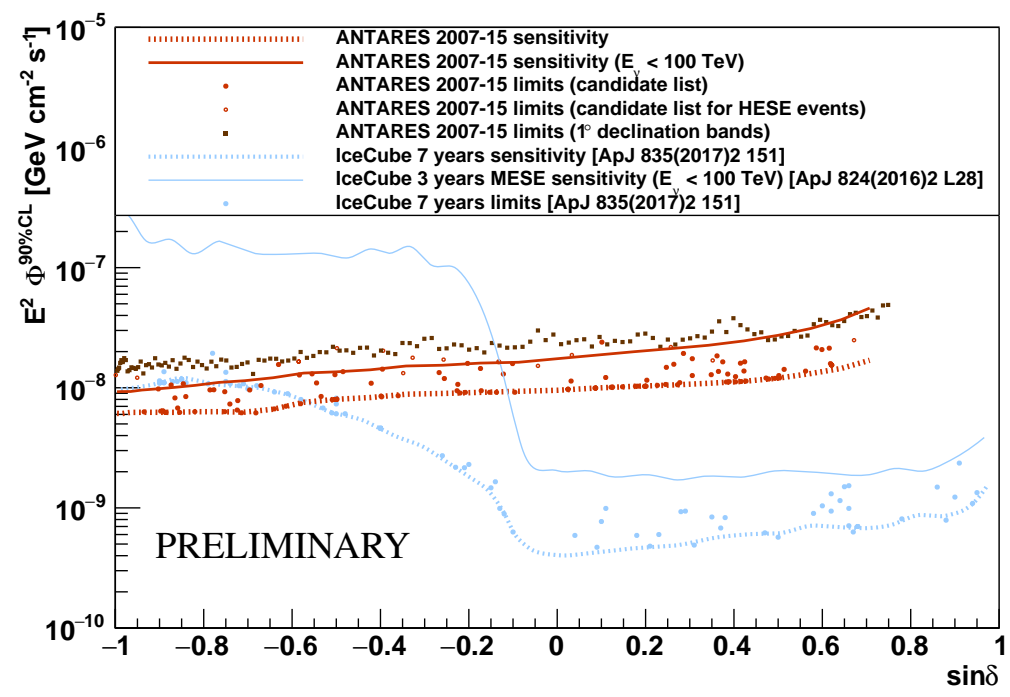

Figure 1: Upper limits at a $90 \%$ C.L. on the total signal flux (sum of the contributions of the three neutrinos flavours) from the investigated candidates assuming an $E^{-2}$ spectrum (red circles). The dashed red line shows the ANTARES sensitivity and the blue dashed line the sensitivity of the seven years point-like source analysis by the IceCube Collaboration for comparison [8]. The upper-limits obtained in this analysis are also included (blue dots). The ANTARES $5 \sigma$ pre-trial discovery flux is a factor 2.5 to 2.9 larger than the sensitivity. The curve for the sensitivity for neutrino energies under $100 \mathrm{TeV}$ is also included (solid red line). The IceCube curve for energies under $100 \mathrm{TeV}$ (solid blue line) is obtained from the 3 years MESE analysis [9]. The limits of the most significant cluster obtained in bands of $1^{\circ}$ in declination (dark red squares) are also shown.

\subsection{Candidate List Search}

In the candidate list search, the directions of a pre-selected list of 106 known astronomical objects, which are promising neutrinos emitters, are investigated to look for an excess of neutrino events. The list of the astronomical candidates along with their equatorial coordinates, fitted number of signal events and upper limits on the flux is shown in Table 1. The most signal-like cluster is found at the location of HESSJ0632+057 at $(\alpha, \delta)=\left(98.24^{\circ}, 5.81^{\circ}\right)$, with a pre-trial p-value of $0.16 \%$. The post-trial significance of the cluster is $13 \%$ or $1.5 \sigma$ (two-sided convention). The sensitivities and limits calculated with the Neyman method at a 90\% C.L. for this search are shown in Figure 1 as a function of the declination.

A separate candidate list search is performed to investigate the 13 IceCube (IC) HESE classified as muon tracks $[10,11,12]$. The non-negligible estimated angular error of these events is accounted for by letting the direction parameters in the likelihood maximisation free to vary around the position of the IC tracks within a cone twice as large as their estimated angular error. In Table 2 the coordinates of these events together with their angular uncertainty (provided by the IceCube 
Collaboration), fitted number of signal events and upper limits on the flux derived from our analysis are shown. The HESE candidate with the largest excess in fitted signal is the IceCube track with ID 3 and $\mu_{\text {sig }}=5.3$. The fitted cluster is located at $(\alpha, \delta)=\left(130.1^{\circ},-29.8^{\circ}\right)$, which is at a distance of $1.5^{\circ}$ from the original HESE track at $(\alpha, \delta)=\left(127.9^{\circ},-31.2^{\circ}\right)$, and has an observed post-trial p-value of 0.20 (1.2 $\sigma$ significance).

\subsection{Galactic Center Region}

A restricted region defined as an ellipse around the GC with semi-axes of $20^{\circ}$ in galactic longitude and $15^{\circ}$ in galactic latitude is scanned to search for clustering of events with respect to the expected background as in the full-sky search (Section 4.1). The motivation of this dedicated search around the GC relies on the number of high-energy neutrino events observed by the IceCube detector $[10,11]$ that appear to cluster in this region. Furthermore, the HESS Collaboration recently discovered an accelerator of $\mathrm{PeV}$ protons in the Galactic Center [13] that could induce neutrinos. Assuming the usual $E^{-2}$ spectrum, the most significant cluster found in this region is located at $(\alpha, \delta)=\left(257.4^{\circ},-41.0^{\circ}\right)$ with a pre-trial p-value of $0.09 \%$ and a post-trial significance of $60 \%$.

Moreover, since a fit of the spectral index to the astrophysical neutrino signal detected by IceCube resulted in $\Phi(E) \propto E^{-\gamma}$ with $\gamma=2.3$ [11] and $\gamma=2.6$ [14], slightly softer spectra than the $E^{-2}$ dependence are tested $(\gamma=2.1,2.3,2.5)$. Different source extensions, quantified by the $\sigma$ of the Gaussian distribution, are also simulated $\left(\sigma=0.5^{\circ}, 1.0^{\circ}, 2.0^{\circ}\right)$. For a spectral index of $\gamma=$ 2.5 and a point source, the most significant cluster is found at $(\alpha, \delta)=\left(273.0^{\circ},-42.2^{\circ}\right)$, with a pre-trial p-value of $0.02 \%$ and a post-trial significance of $30 \%$. The most significant clusters for the remaining spectral indices and source extensions are located within $1^{\circ}$ from the latter. In Figure 2, the declination-dependent limit of this search is shown both for different energy spectral indices $\gamma$ and different source extensions $\sigma$.
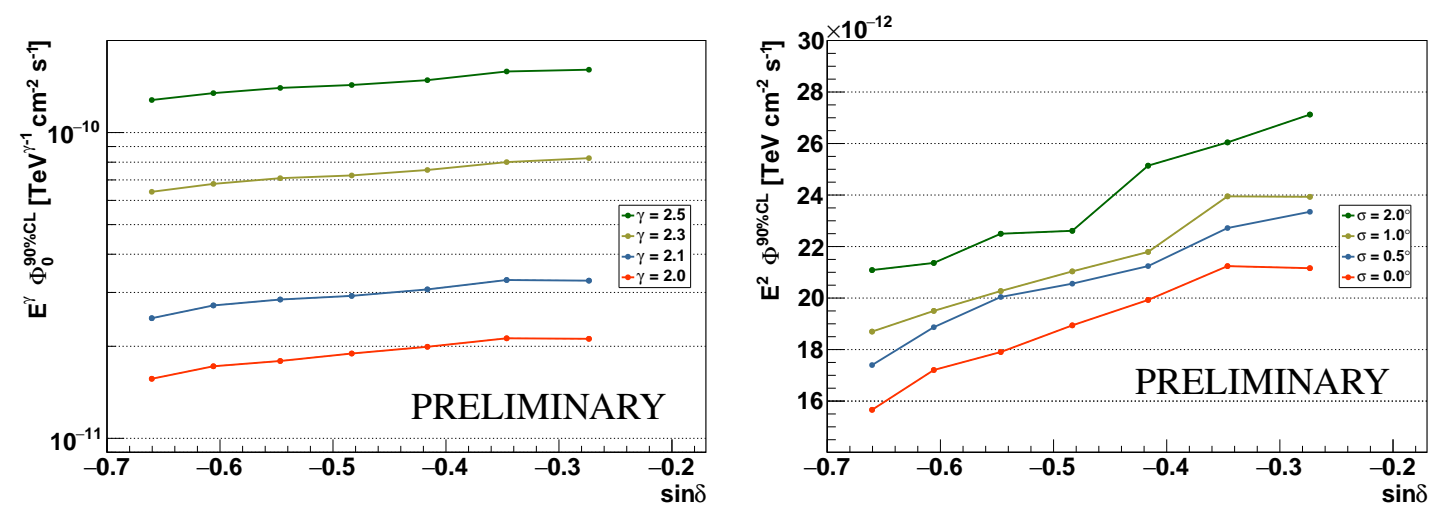

Figure 2: $90 \%$ C.L. upper limits of a search restricted to the region around the origin of the galactic coordinates at $(\alpha, \delta)=\left(266.40^{\circ},-28.94^{\circ}\right)$ assuming different spectral indices for the neutrino flux (left) and different source extensions for $\gamma=2$ (right).

\subsection{Sagittarius A*}

The super-massive black hole Sagittarius $A^{*}$, located at $(\alpha, \delta)=\left(266.42^{\circ},-29.01^{\circ}\right)$, is a strong candidate for cosmic neutrino production [15]. It is investigated as an extended source 


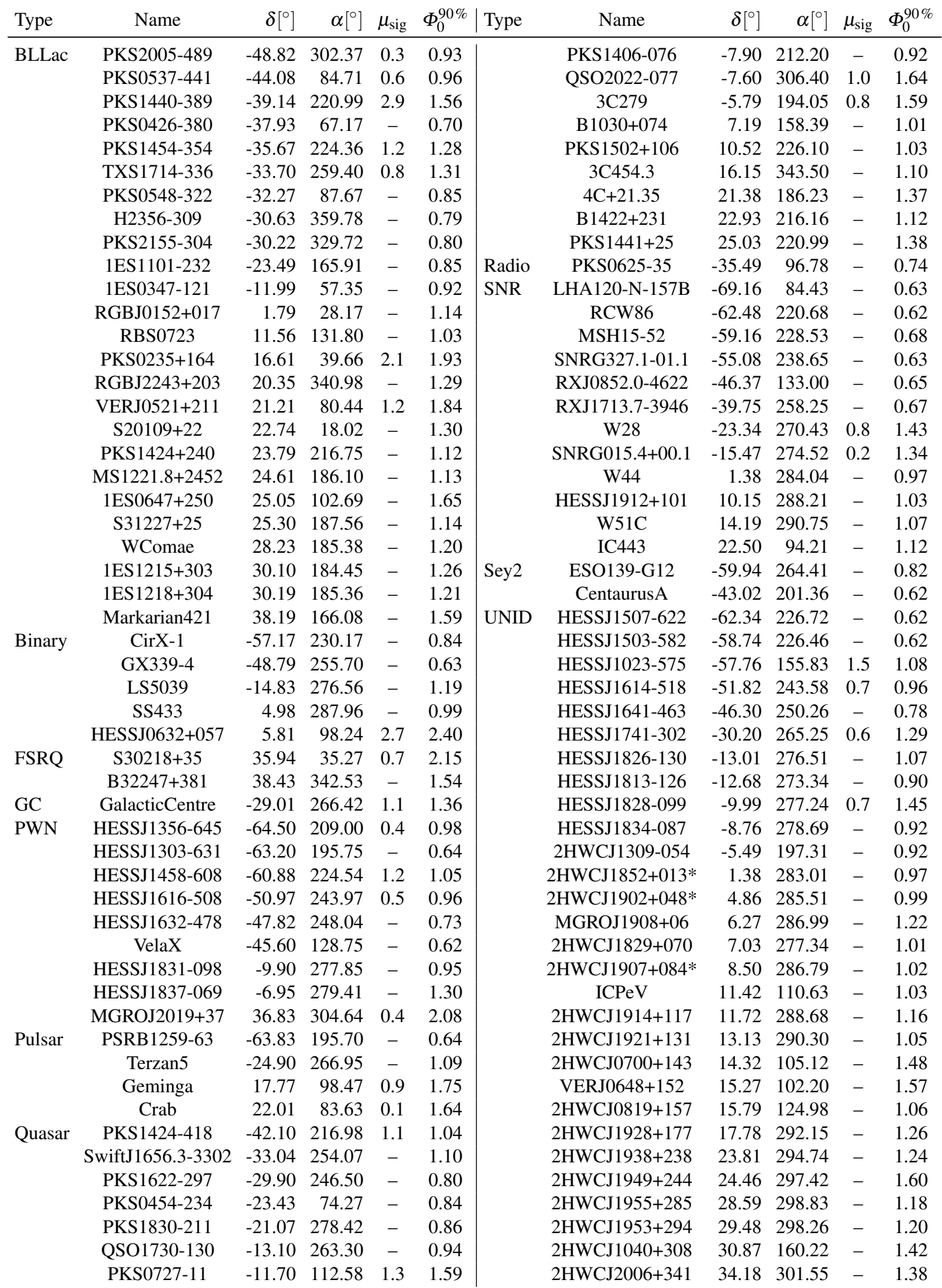

Table 1: List of astrophysical objects used in the candidate list search. Presented are the object's coordinates in declination $(\delta)$ and right-ascension $(\alpha)$. The first column reports the type of source: Binary means X-Ray binary, GC means Galactic Center, Radio means Radio Galaxy, Sey2 means Seyfert 2 Galaxy, UNID means unidentified. The last two columns show the sum of the fitted number of signal track and shower events $\mu_{\text {sig }}=\mu_{\text {sig }}^{t r}+\mu_{\text {sig }}^{s h}$, and the $90 \%$ C.L. upper limits on the total neutrino flux normalization factor $\Phi_{0}^{90 \%}$ (in units of $10^{-8} \mathrm{GeV} \mathrm{cm}^{-2} \mathrm{~s}^{-1}$ ). Candidates of the same type are sorted by declination. 


\begin{tabular}{crrrrr|rrrrrr} 
HESE ID & $\delta\left[^{\circ}\right]$ & $\alpha\left[^{\circ}\right]$ & $\beta_{\mathrm{IC}}\left[^{\circ}\right]$ & $\mu_{\text {sig }}$ & $\Phi_{0}^{90 \%}$ & HESE ID & $\delta\left[^{\circ}\right]$ & $\alpha\left[^{\circ}\right]$ & $\beta_{\mathrm{IC}}\left[^{\circ}\right]$ & $\mu_{\text {sig }}$ & $\Phi_{0}^{90 \%}$ \\
\hline 3 & -31.2 & 127.9 & 1.4 & 5.4 & 2.1 & 37 & 20.7 & 167.3 & 1.2 & 0.5 & 1.7 \\
5 & -0.4 & 110.6 & 1.2 & 0.6 & 1.5 & 38 & 14.0 & 93.3 & 1.2 & 1.0 & 2.1 \\
8 & -21.2 & 182.4 & 1.3 & 1.3 & 1.7 & 43 & -22.0 & 206.6 & 1.3 & 0.3 & 1.3 \\
13 & 40.3 & 67.9 & 1.2 & 0.8 & 2.4 & 44 & 0.0 & 336.7 & 1.2 & 1.8 & 1.8 \\
18 & -24.8 & 345.6 & 1.3 & 1.7 & 2.0 & 45 & -86.3 & 219.0 & 1.2 & 1.6 & 1.2 \\
23 & -13.2 & 208.7 & 1.9 & 0.9 & 1.7 & 53 & -37.7 & 239.0 & 1.2 & 3.1 & 1.6 \\
28 & -71.5 & 164.8 & 1.3 & 1.6 & 1.2 & & & & & &
\end{tabular}

Table 2: The 13 IceCube HESE muon track candidates $[10,12]$ that are in the field of view of the ANTARES detector. The table gives the equatorial coordinates, the angular error estimate $\beta_{\mathrm{IC}}$ of the event, the sum of the fitted number of signal track and shower events $\mu_{\text {sig }}=\mu_{\text {sig }}^{t r}+\mu_{\text {sig }}^{s h}$ and the $90 \%$ C.L. upper limits on flux $\Phi_{0}^{90 \%}$ (in units of $10^{-8} \mathrm{GeV} \mathrm{cm}^{-2} \mathrm{~s}^{-1}$ ).

with widths between $0.5^{\circ}$ and $5^{\circ}$. Indeed, the high concentration of candidate sources in the GC region makes it more likely to detect an extended signal before identifying individual point-like sources. The sensitivity and upper limits obtained for the assumption of different source extensions are shown in Figure 3. The largest excess above the background is found for no extension with a pre-trial p-value of 0.22 .

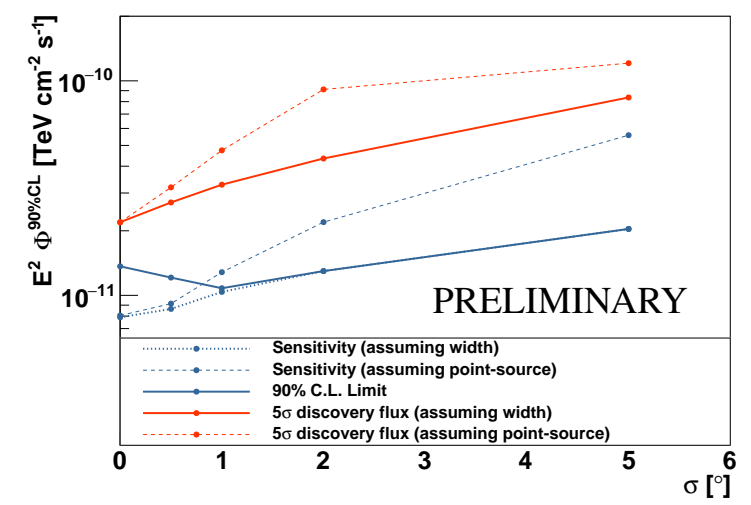

Figure 3: Discovery flux (solid red), median sensitivity (dotted blue) and $90 \%$ C.L. upper limits (solid blue) for a search for an extended source at Sagittarius $A^{*}$ at $(\alpha, \delta)=\left(266.42^{\circ},-29.01^{\circ}\right)$ assuming different angular extensions $\sigma$. The dashed lines correspond to the point-like source assumption.

\section{Conclusions}

In this proceeding, the results of the first point-like source search that exploits the combined information from the track and shower events detected by the ANTARES telescope have been presented. Four different searches for cosmic neutrino sources have been performed: a scan over the whole ANTARES visible sky, an investigation of 106 astrophysical candidates and 13 IceCube muon tracks, a dedicated analysis of the GC region and a study of Sagittarius A* investigated as a possible extended source. No significant evidence of cosmic neutrino sources has been found. Nevertheless, these searches provide the most sensitive limits for a large fraction of the Southern Sky, especially at neutrino energies below $100 \mathrm{TeV}$. A future joint analysis of the ANTARES events 
with data from the IceCube detector could significantly improve the sensitivity of the point-like source in the Southern sky, as already shown in the past [16].

\section{References}

[1] M. Ageron et al. (ANTARES collaboration), ANTARES: the first undersea neutrino telescope, Nucl.Instrum.Meth. A656 (2011) 11-38 [arXiv:1104.1607]

[2] T. Michael, Neutrino point source search including cascade events with the ANTARES neutrino telescope, in proceedings of 34th International Cosmic Ray Conference (ICRC 2015), POS ICRC2015 (2016) 1078

[3] A. Albert et al. (ANTARES collaboration), First all-flavor Neutrino Point-like Source Search with the ANTARES Neutrino Telescope, [arXiv:1706.01857]

[4] S. Adrian-Martínez et al. (ANTARES collaboration), Search for Cosmic Neutrino Point Sources with Four Years of Data from the ANTARES Telescope, Astrophys.J. 760 (2012) 53 [arXiv:1207.3105]

[5] F. Schüssler, Energy reconstruction in neutrino telescopes, in proceedings of 33rd International Cosmic Ray Conference (ICRC 2013)

[6] S. Adrian-Martínez et al. (ANTARES collaboration), Measurement of the atmospheric $v_{\mu}$ energy spectrum from $100 \mathrm{GeV}$ to $200 \mathrm{TeV}$ with the ANTARES telescope, Eur.Phys.J. C73 (2013) 2606 [arXiv:1308.1599]

[7] J. Neyman, Outline of a Theory of Statistical Estimation Based on the Classical Theory of Probability, Phil.Trans.Roy.Soc.Lond. A236 (1937) no.767, 333-380

[8] M. G. Aartsen et al. (IceCube collaboration), All-sky search for time-integrated neutrino emission from astrophysical sources with 7 years of IceCube data, Astrophys.J. 835 (2017) no.2, 151 [arXiv:1609.04981]

[9] M. G. Aartsen et al. (IceCube collaboration), Lowering IceCube's Energy Threshold for Point Source Searches in the Southern Sky, Astrophys.J. 824 (2016) no.2, L28 [arXiv:1605.00163]

[10] M. G. Aartsen et al. (IceCube collaboration), Observation of High-Energy Astrophysical Neutrinos in Three Years of IceCube Data, Phys.Rev.Lett. 113 (2014) 101101 [arXiv:1405.5303]

[11] M. G. Aartsen et al. (IceCube collaboration), Searches for Extended and Point-like Neutrino Sources with Four Years of IceCube Data, Astrophys.J. 796 (2014) no.2, 109 [arXiv:1406.6757]

[12] C. Kopper et al., Observation of Astrophysical Neutrinos in Four Years of IceCube Data, in proceedings of 34th International Cosmic Ray Conference (ICRC 2015), POS ICRC2015 (2016) 1081

[13] A. Abramowski et al. (H.E.S.S collaboration), Acceleration of petaelectronvolt protons in the Galactic Centre, Nature 531 (2016) 476 [arXiv:1603.07730]

[14] The IceCube Collaboration, Observation of Astrophysical Neutrinos in Four Years of IceCube Data, in proceedings of 34th International Cosmic Ray Conference (ICRC 2015), POS ICRC2015 (2016) 1081

[15] Y. Bai et al., Neutrino lighthouse at Sagittarius A*, Phys.Rev. D90 (2014) no.6, 063012 [arXiv:1407.2243]

[16] S. Adrián-Martínez et al. (ANTARES and IceCube collaborations), The First Combined Search for Neutrino Point-sources in the Southern Hemisphere With the Antares and Icecube Neutrino Telescopes , Astrophys.J. 823 (2016) no.1, 65 [arXiv:1511.02149] 